\title{
Fatores associados à adesão a um programa de tratamento de alcoolistas
}

\author{
Factors associated with adherence in a alcoholic program \\ Mário Sérgio Ribeiro', Luiz Cláudio Ribeiro², Marcondes Antunes Garcia33, Grazielle Fialho de Souza33, \\ Karolina Danielle Carvalho de Sousa ${ }^{3}$, Rachel Bueno Nogueira ${ }^{3}$
}

\section{RESUMO}

Objetivo: A medida da adesão tem sido considerada alternativa objetiva e versátil para avaliação do resultado do tratamento de alcoolistas. Este estudo avaliou fatores associados à adesão de alcoolistas atendidos em um programa ambulatorial. Método: Foram estudados 300 alcoolistas que concluíram a fase de avaliação do programa e avaliada a associação da adesão dos pacientes ao tratamento a todas as mais de mil variáveis do banco de dados do programa, utilizando o teste qui-quadrado de Pearson $(p<0,1)$. Resultados: Entre as variáveis que se associaram positivamente à adesão foram identificadas: ter filhos; relação conjugal estável; afirmar problemas psicológicos; ter sofrido esquecimento ou fraqueza recentemente; sentir-se irritado quando alcoolizado; beber sozinho; apresentar comorbidade psiquiátrica; já ter procurado tratamento para alcoolismo, tratamento em psiquiatria; uso anterior de antidepressivos etc. Associaram-se negativamente: reduzir consumo por influência familiar; sentir-se auto-suficiente, expansivo, insatisfeito quando sóbrio; sentir-se auto-suficiente ou conformado quando alcoolizado; início do consumo com idade acima

Palavras-chave

Alcoolismo, adesão, avaliação de alcoolistas, diagnóstico. da média do grupo; ter-se envolvido em agressão física com amigos etc. Conclusão: Os resultados sugerem a necessidade de desenvolvimento de estratégias diferenciadas para o cuidado de determinados grupos de pacientes e contribuem para a simplificação das rotinas de avaliação de alcoolistas.

\section{ABSTRACT}

Objective: The evaluation of adherence to treatment has been considered an objective and versatile alternative to alcoholism treatment result assessment. The purpose of this study is to identify factors associated with adherence to an outpatient alcoholism treatment program. Method: This study included 300 alcoholic patients that concluded the program assessment stage. Crosstabs were performed to verify the association of adherence to treatment on all other (more than 1,000) variables in the data bank. Statistical significance was given by Pearson's Quisquare test $(p<0.1)$. Results: Among variables with a positive association to adherence were:

Laboratório de Pesquisas em Personalidade, Álcool e Drogas (LAPPDA-UFJF) do Departamento de Clínica Médica da Faculdade de Medicina da Universidade Federal de Juiz de Fora (UFJF).

1 Psiquiatria da Faculdade de Medicina da Universidade Federal de Juiz de Fora (UFJF).

2 Departamento de Estatística da UFJF

3 Curso de Medicina da UFJF

Endereço para correspondência: Mário Sérgio Ribeiro

Rua Severino Meireles, 325/902 - 36025-040 - Juiz de Fora, MG

E-mail:mariosribeiro@acessa.com 


\section{Keywords}

Alcoholism, adherence, alcoholics assessment, diagnosis. to have children and stable matrimonial relationship; affirm psychological problems; lately noticed forgetfulness and weakness; felt irritability when drunk, consuming it alone; present a psychiatric comorbidity; had already looked for alcoholism treatment, got help from AA, psychiatric treatment and made use of antidepressants; etc. Negative associations were: reduced consumption due to family influence; felt self-sufficiency, expansive and unsatisfied while sober; self-sufficient or resigned when under alcohol; first consumptions above group's average age; involved in physical aggression with friends; etc. Conclusions: These findings suggest the need of developing particular therapeutic strategies to address specific groups of patients and also contribute to the simplification of alcoholism assessment routines.
Em ambientes de pesquisa, é freqüente que as avaliações de resultado sejam realizadas após períodos relativamente breves, o que resulta grupos de sujeitos com o mesmo padrão de adesão ao tratamento. Em geral, a avaliação dos resultados dos tratamentos de alcoolismo se faz por meio de: a) variáveis cognitivo-comportamentais, de atitude e personalidade; b) variáveis relacionadas ao impacto no comportamento de beber; c) variáveis relacionadas ao funcionamento de várias áreas da vida do paciente. Para cada indicador de resultado considerado têm sido desenvolvidos instrumentos específicos de avaliação, cujo uso costuma ser comple$\mathrm{xo}$, requerendo disponibilidade de tempo, treinamento e recursos específicos'.

Para a avaliação de programas assistenciais rotineiros de tratamento do alcoolismo - em geral, de mais longa duração -, o uso de múltiplos critérios e indicadores não se cerca da necessária praticidade. Segundo Connors et al. ${ }^{2}$, as baixas taxas de adesão ao tratamento são problemas extensivos e comuns em alcoolismo. Corrao et al. ${ }^{3}$, responsáveis pelo projeto ASSALT (lessons from the italian assessment of alcoholism treatment), identificaram alto risco de abandono por parte dos pacientes que não foram capazes de manter abstinência - não um lapso, isolado, mas recaídas recorrentes. Segundo os pesquisadores italianos, a medida da adesão constituiria, por conseguinte, uma medida realista e acurada para a avaliação do resultado do tratamento, alternativamente à verificação de comportamentos ligados à ingesta - de mais difícil mensuração .

Diversos fatores associados à adesão aos programas terapêuticos já foram relativamente identificados. Ainda que diferentes estudos não tenham conseguido chegar a conclusões consistentes e definitivas, características já associadas à adesão ao tratamento de alcoolistas incluem: o envolvimento de familiares ou pessoa próxima e significativa ${ }^{4}$; ordenação judicial ${ }^{5}$; gênero ${ }^{6}$; idade ${ }^{7}$; uso de outras substâncias psicoativas e psicopatologia comórbida ${ }^{8,9}$; intensidade e duração do tratamento ${ }^{10,11}$. Aparentemente, descrença na gravidade clínica das conseqüências do uso de álcool pode resultar a falta de adesão ao tratamento ${ }^{12}$. Por sua vez, prévio uso de técnicas motivacionais ${ }^{2}$ pode levar alcoolistas a aderirem mais e manterem-se abstinentes por período mais longo. Em última instância, o conhecimento de características dos pacientes que interfiram em sua adesão aos programas terapêuticos contribui para a adequação deles a necessidades específicas, melhorando a eficácia do tratamento.

Consideradas as conclusões do projeto ASSALT, o objetivo deste estudo é uma análise exploratória de características sociodemográficas e clínicas possivelmente associadas à adesão de alcoolistas atendidos pelo Programa de Atenção a Dependentes Químicos (PADQ) do Sistema Municipal de Saúde Mental de Juiz de Fora (SMSM-JF). O tempo de permanência dos pacientes em contato com as rotinas terapêuticas propostas foi a variável de resultado avaliada no estudo.

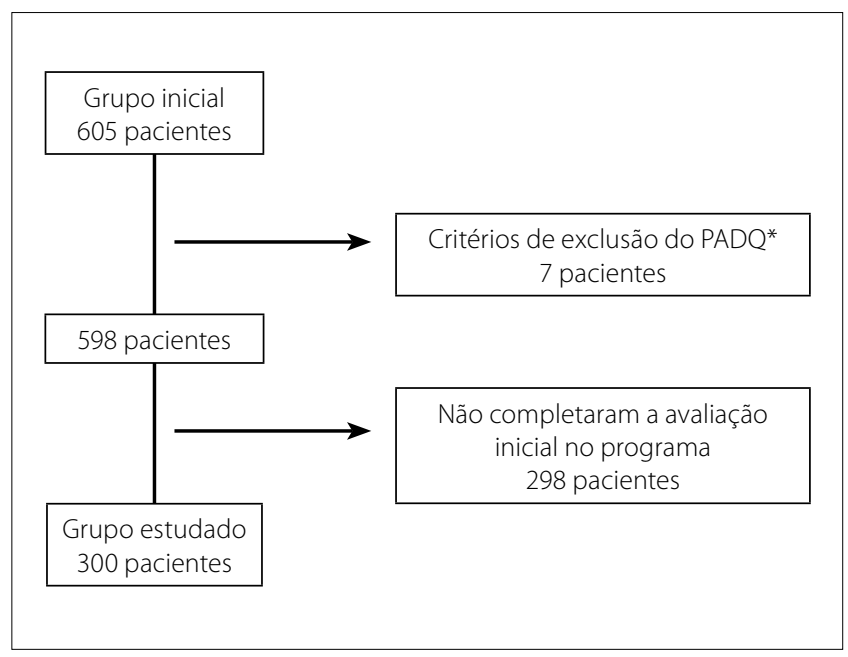

* Os critérios de exclusão do PADQ do Sistema Único de Saúde de Juiz de Fora (SUS-JF) incluíam alcoolistas com diagnósticos de F10.1, F10.6, F10.73 e F10.74.

Figura 1. Síntese do processo de composição do grupo estudado 


\section{MÉTODOS}

\section{Delineamento do estudo}

Partiu-se de experimento natural, observacional, sem intervenção do grupo de pesquisadores na alocação dos sujeitos ou procedimentos terapêuticos. O PADQ era um serviço de referência para tratamento de dependentes de álcool e/ou outras substâncias psicoativas de Juiz de Fora, município com mais de 500 mil habitantes da Zona da Mata mineira. As avaliações e as intervenções eram realizadas por profissionais de distintas formações - enfermagem, medicina (clínico-geral e psiquiatras), psicologia e serviço social, e os diagnósticos relativos ao alcoolismo e comorbidades psiquiátricas foram realizados em base clínica, seguindo critérios da CID-10.

Esta pesquisa foi aprovada pelos comitês de ética do Hospital Universitário da Universidade Federal de Juiz de Fora (HU-UFJF) e da Universidade Federal de juiz de Fora, e todos os indivíduos assinaram termo de consentimento e esclarecido antes de qualquer procedimento do estudo fosse iniciado.

\section{População}

O estudo incluiu pacientes com primeiro atendimento entre outubro de 1997 e dezembro de 2004, tendo sido avaliados 605 adultos com transtornos mentais e/ou do comportamento associados ao uso apenas de alcoólicos. Dependência à nicotina não foi considerada, nem como critério de inclusão nem de exclusão.

A rotina de avaliação multiprofissional do PADQ - prevista para ser realizada em aproximadamente quatro semanas - compunha-se de entrevistas estruturadas e semi-estruturadas, exames clínico e psiquiátrico. As entrevistas semi-estruturadas envolviam: aspectos demográficos e sociais; padrões quantitativo e qualitativo relativos ao consumo; identificação de estados emocionais e outros fenômenos associados ao consumo ou à sobriedade; substâncias consumidas; repercussões macro e microssociais; e atitudes do paciente diante de sua problemática. Em seu conjunto, as avaliações realizadas no PADQ eram registradas em um banco de dados criado com o programa Epi-Info 6.04. A rotina assistencial do PADQ já foi apresentada e discutida anteriormente ${ }^{9}$.

Conforme sintetizado na Figura 1, foram retirados das análises aqui realizadas sete pacientes que se enquadravam nos critérios de exclusão do próprio programa assistencial nomeadamente, uso nocivo, síndrome amnéstica, demência e outros comprometimentos cognitivos persistentes decorrentes do uso de alcoólicos - e 298 usuários que não completaram a avaliação inicial. Não foram excluídos do estudo pacientes que apresentassem quaisquer comorbidades psiquiátricas. No total foram, portanto, excluídos 305 sujeitos e o grupo estudado ficou composto por 300 pacientes.

\section{Variáveis}

As variáveis utilizadas decorrem diretamente das mais de mil variáveis constantes do banco de dados do PADQ ou geradas por combinações destas. Tais combinações se restringiram ao cálculo da média de variáveis quantitativas e a recodificações, objetivando transformar variáveis policategóricas em binárias, agrupando cada categoria por semeIhança ou de modo que separasse os sujeitos da forma mais homogênea e/ou lógica possível. Como exemplos podem ser citadas as variáveis estado civil (com relação estável ou sem relação estável), escolaridade (até primeiro grau completo ou acima do primeiro grau completo) e renda pessoal (até um salário mínimo ou acima de um salário mínimo). O tempo de permanência dos pacientes em tratamento foi recodificado em adesão até seis meses e adesão superior a seis meses (ou, simplesmente, adesão superior).

\section{Análise estatística}

Com o objetivo de não limitar o estudo por quaisquer critérios a priori, procedeu-se ao cruzamento entre todas as variáveis que compunham o banco de dados com a variável adesão dos pacientes ao programa terapêutico, utilizando-se para tanto a recodificação binária referida anteriormente. A associação de cada variável à adesão ao tratamento foi verificada por meio do teste qui-quadrado de Pearson - utilizado também para testar as diferenças entre os grupos de pacientes que concluíram ou não a fase de avaliações. Para aumentar a confiabilidade dos resultados, não foram considerados os cruzamentos que resultaram menos de cinco sujeitos em qualquer casela. Uma vez que se trata de estudo exploratório, optou-se por incluir, na apresentação dos resultados, não somente os cruzamentos estatisticamente significantes ( $p$ valor $<0,05$ ) pelo teste qui-quadrado, como também os resultados marginalmente significantes $(0,1>p \geq 0,05)$.

\section{RESULTADOS}

Os aspectos demográficos e socioeconômicos dos sujeitos incluídos e excluídos da análise estão apresentados na Tabela 1. Ainda que para o conjunto de pacientes que não completou a fase de avaliações do PADQ se tenha observado mais elevado percentual de missing (não-resposta) nas diversas variáveis, a avaliação dos dois grupos revelou algumas diferenças estatisticamente significante entre eles. As características dos pacientes que, significativamente, se associaram a maior conclusão da avaliação foram: não ter nascido em Juiz de Fora ( $p=0,041)$; escolaridade acima da média do grupo ( $p=0,052)$; professar a religião católica $(p=0,004)$; freqüentar o culto religioso $(p=0,032)$; e renda familiar abaixo da média do grupo $(p=0,000)$. 
Tabela 1. Variáveis sociodemográficas dos sujeitos que concluíram e que não concluíram a avaliação.

\begin{tabular}{|c|c|c|c|}
\hline \multirow[b]{2}{*}{ Variáveis } & \multicolumn{2}{|c|}{ Sujeitos } & \multirow[b]{2}{*}{ p valor* } \\
\hline & $\begin{array}{l}\text { Concluíram a avaliação } \\
\qquad(\mathrm{n}=300)(\%)^{\prime}\end{array}$ & $\begin{array}{l}\text { Não concluíram a avaliação } \\
\qquad(\mathrm{n}=298)(\%)^{*}\end{array}$ & \\
\hline \multicolumn{4}{|l|}{ Demográficas } \\
\hline Sexo masculino & 88,9 & 90,0 & 0,695 \\
\hline Corbranca & 67,1 & 63,1 & 0,392 \\
\hline Idade média $( \pm \mathrm{DP})^{\sharp \#}$ & $42,35(8,66)$ & $39,78(9,01)$ & 0,659 \\
\hline Não naturais de Juiz de Fora & 55,0 & 44,9 & $0,041^{* *}$ \\
\hline \multicolumn{4}{|l|}{ Sociais } \\
\hline Baixa escolaridade ${ }^{\# \#}$ & 79,9 & 87,2 & $0,052^{* *}$ \\
\hline Casados & 50,9 & 46,4 & 0,360 \\
\hline Se casado, é o primeiro relacionamento conjugal & 71,5 & 71,8 & 0,965 \\
\hline Tem filhos & 74,7 & 73,5 & 0,769 \\
\hline Tem até 2 filhos & 58,8 & 62,2 & 0,544 \\
\hline Tem até 4 moradores na residência & 66,3 & 63,8 & 0,592 \\
\hline Religião católica & 84,8 & 73,5 & $0,004^{* *}$ \\
\hline Freqüentam 0 culto & 61,4 & 50,4 & $0,032^{* *}$ \\
\hline \multicolumn{4}{|l|}{ Econômicas } \\
\hline Não trabalham na profissão & 58,5 & 54,5 & 0,410 \\
\hline Desempregados ou recebendo benefício & 65,5 & 71,1 & 0,229 \\
\hline \multicolumn{4}{|l|}{ Ocupacionais } \\
\hline Dependem economicamente de outrem & 50,2 & 50,3 & 0,973 \\
\hline Não são arrimo de família & 74,9 & 75,2 & 0,953 \\
\hline Renda pessoal até um SM"\#\#\# & 49,8 & 71,7 & 0,709 \\
\hline Renda familiar de um a três SM & 55,4 & 46,0 & $0,000^{* *}$ \\
\hline
\end{tabular}

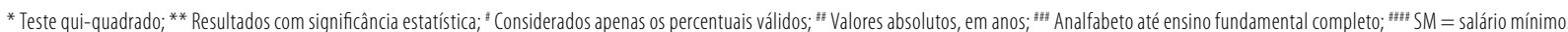

Com o objetivo de simplificar a apresentação dos resultados, a Tabela 2 apresenta, de maneira sucinta, as correlações significativas ou, ao menos, marginalmente significativas pelo teste qui-quadrado. Nele, as variáveis foram agrupadas por suas características predominantes sociodemográficas; sintomatológicas; ligadas a sentimentos, comportamentos etc. - e foram registrados apenas os percentuais relativos aos grupos de sujeitos com padrão de adesão considerado superior, isto é, igual ou maior que seis meses.

Como exemplo da interpretação dos resultados, remetese ao posicionamento dos pacientes diante de duas características sociodemográficas, isto é, às suas respostas ante a questão de ter ou não filhos e considerar-se ou não um profissional autônomo. O fato de os pacientes terem filhos influenciou positivamente a adesão: $58,3 \%$ dos pacientes que responderam de modo afirmativo à pergunta alcançaram adesão superior, ao passo que apenas 38,4\% do subgrupo que não tinha filhos alcançaram este padrão de adesão ( $p=0,003)$; considerados todos os 289 pacientes que responderam a essa questão, o percentual de adesão superior foi de 53,3\%. Quanto a se considerar ou não profissional autônomo, a resposta negativa influenciou positivamente a adesão: 57,1\% dos pacientes que não se consideravam autônomos alcançaram o padrão de adesão considerado superior, enquanto este resultado foi alcançado apenas por 44,6\% dos sujeitos que afirmaram serem autônomos; tomados todos os 179 que responderam a essa pergunta, o percentual de adesão superior foi de 52,0\% ( $p=0,098)$.

\section{Características demográficas e sociais}

Ter filhos, manter relação conjugal estável, tempo médio de união acima da média do grupo, estar trabalhando no momento da avaliação e receber mais do que um salário mínimo foram variáveis associadas positivamente à adesão superior. Considerar-se trabalhador autônomo associou-se negativamente à adesão dos pacientes.

\section{Crenças e influências de terceiros}

Variáveis com associação positiva à adesão foram achar que estava com problemas psicológicos, afirmar saber por que consumia alcoólicos e seu patrão ter sido o primeiro a identificar seu problema com o álcool. Achar que precisava desintoxicar-se, responder que seus familiares eram indiferentes a seu consumo de alcoólicos e responder que já teria reduzido o consumo por influência da família foram respostas associadas negativamente à adesão.

\section{Sintomatologias física e mental}

Sofrer de esquecimento, dor abdominal, edema nos pés, fraqueza ou desmaios nos 30 dias que precederam a avaliação, sentir tremores se não bebia no horário habitual e sentir "algo" se não bebia no horário habitual foram respostas positivamente associadas à adesão superior. Pressão arterial sistólica > $130 \mathrm{mmHg}$ e diastólica $\geq 90 \mathrm{mmHg}$, ter apresentado roncos à ausculta respiratória e afirmar sentir-se inapetente se não bebia no horário habitual se associaram negativamente à adesão superior. 
Tabela 2. Características associadas à adesão superior (igual ou maior que 6 meses) ao tratamento $(n=300)^{*}$.

\begin{tabular}{|c|c|c|c|c|}
\hline \multirow[t]{2}{*}{ Características } & \multicolumn{2}{|c|}{$\begin{array}{l}\text { Respostas dos sujeitos } \\
\text { com adesão superior }\end{array}$} & \multirow{2}{*}{$\begin{array}{l}\text { Adesão superior } \\
\text { em todo o grupo } \\
(\%)\end{array}$} & \multirow[t]{2}{*}{ pvalor } \\
\hline & Não(\%) & $\operatorname{Sim}(\%)$ & & \\
\hline \multicolumn{5}{|l|}{ Demográficas e sociais } \\
\hline Tinha filhos? $(n=289)$ & 38,4 & 58,3 & 53,3 & $0,003^{* *}$ \\
\hline Mantinha relação conjugal estável? ( $n=293)$ & 47,2 & 59,7 & 53,6 & $0,032^{* *}$ \\
\hline Tinha tempo médio de união acima da média do grupo $(15,1$ anos $) ?(n=180)$ & 50,0 & 68,2 & 58,9 & $0,013^{* *}$ \\
\hline Estava trabalhando no momento da avaliação? $(n=290)$ & 50,5 & 61,0 & 54,1 & 0,089 \\
\hline Recebia mais que um salário mínimo? $(\mathrm{n}=287)$ & 48,3 & 59,0 & 53,7 & 0,067 \\
\hline Considerava-se autônomo? $(\mathrm{n}=179)$ & 57,1 & 44,6 & 52,0 & 0,098 \\
\hline \multicolumn{5}{|l|}{ Crenças e influências de terceiros } \\
\hline Achava que precisava desintoxicar-se? $(n=282)$ & 57,9 & 47,5 & 54,3 & 0,093 \\
\hline Achava que estava com problemas psicológicos? $(n=282)$ & 50,5 & 63,4 & 54,3 & $0,048^{* *}$ \\
\hline Sabia por que consumia álcool? $(n=282)$ & 52,5 & 76,2 & 54,3 & $0,036^{* *}$ \\
\hline Quem primeiro identificou o problema com álcool foi o patrão? $(n=282)$ & 52,9 & 76,2 & 54,6 & $0,039^{* *}$ \\
\hline Familiares eram indiferentes ao consumo de álcool do paciente? $(n=284)$ & 56,2 & 29,4 & 54,6 & $0,032^{* *}$ \\
\hline Reduziu o consumo por influência da família? $(n=75)$ & 61,2 & 30,8 & 50,7 & $0,012^{* *}$ \\
\hline \multicolumn{5}{|l|}{ Sintomatológicas } \\
\hline Nos 30 dias que precederam à avaliação, sofreu de esquecimento? $(n=259)$ & 46,2 & 60,0 & 53,7 & $0,027^{* *}$ \\
\hline Nos 30 dias que precederam à avaliação, sofreu de dor abdominal? $(n=261)$ & 48,4 & 66,7 & 53,6 & $0,007^{* *}$ \\
\hline Nos 30 dias que precederam à avaliação, apresentou edema nos pés? $(n=261)$ & 51,4 & 65,9 & 53,6 & 0,088 \\
\hline Nos 30 dias que precederam à avaliação, sofreu de fraqueza? $(n=260)$ & 47,4 & 62,5 & 53,5 & $0,017^{* *}$ \\
\hline Nos 30 dias que precederam à avaliação, apresentou desmaios? $(n=259)$ & 50,5 & 63,9 & 53,7 & 0,066 \\
\hline A pressão arterial sistólica era maior que 130 mmHg? $(n=261)$ & 59,5 & 47,2 & 54,4 & $0,050^{* *}$ \\
\hline A pressão arterial diastólica era igual ou maior que 90 mmHg? $(n=261)$ & 58,9 & 47,6 & 54,4 & 0,074 \\
\hline Apresentou roncos à ausculta respiratória? $(n=192)$ & 58,7 & 32,0 & 55,2 & $0,012^{* *}$ \\
\hline Se não bebia no horário habitual sentia-se inapetente? ( $n=266)$ & 59,3 & 46,8 & 55,6 & 0,063 \\
\hline Se não bebia no horário habitual sentia tremores? $(n=266)$ & 45,3 & 59,7 & 55,6 & $0,034^{* *}$ \\
\hline Alguma reação desagradável acontecia se não bebia no horário habitual? $(\mathrm{n}=266)$ & 39,1 & 57,2 & 55,6 & 0,095 \\
\hline \multicolumn{5}{|l|}{ Comportamento e padrão de consumo } \\
\hline Começou a beber com idade acima da média do grupo $(16,4$ anos)? $(n=268)$ & 60,9 & 45,8 & 54,9 & $0,015^{* *}$ \\
\hline Quando começou a beber, bebia sozinho? $(n=269)$ & 51,0 & 65,3 & 55,0 & $0,034^{* *}$ \\
\hline Quando começou a beber, bebia durante 0 trabalho? $(n=269)$ & 53,3 & 72,0 & 55,0 & 0,073 \\
\hline Quando aumentou o consumo, bebia sozinho? $(n=266)$ & 49,5 & 60,1 & 56,0 & 0,090 \\
\hline Quando aumentou o consumo, bebia com colegas de trabalho? $(n=267)$ & 50,7 & 61,6 & 55,8 & 0,074 \\
\hline Quando aumentou o consumo, bebia para aliviar ou enfrentar situações desagradáveis $(n=267)$ & 50,0 & 61,6 & 55,4 & 0,057 \\
\hline Já tinha se envolvido em agressão física com amigos? $(n=83)$ & 64,2 & 31,3 & 57,8 & $0,017^{* *}$ \\
\hline Costumava dirigir alcoolizado? $(n=115)$ & 37,7 & 63,0 & 47,8 & $0,008^{* *}$ \\
\hline À avaliação, referiu beber mais que 98 doses semanais da bebida principal? $(n=250)$ & 51,4 & 63,4 & 56,8 & 0,058 \\
\hline À avaliação, o consumo total semanal era maior que 154 doses? $(n=251)$ & 51,4 & 63,1 & 56,6 & 0,065 \\
\hline À avaliação, referiu que costumava beber freqüentemente sozinho? $(n=280)$ & 39,5 & 60,8 & 54,6 & $0,001^{* *}$ \\
\hline À avaliação, referiu que costumava beber freqüentemente com amigos? $(n=280)$ & 59,9 & 48,4 & 54,6 & 0,056 \\
\hline \multicolumn{5}{|l|}{ Diagnóstico } \\
\hline Apresentou o critério 4 para dependência do DSM-IV (desejo persistente ou esforços para & & & & \\
\hline reduzir ou controlar uso da substância)? $(n=246)$ & 46,3 & 62,0 & 56,9 & $0,019 * *$ \\
\hline $\begin{array}{l}\text { Apresentou o critério } 6 \text { para dependência do DSM-IV (abandono ou redução de importantes } \\
\text { atividades sociais, ocupacionais ou recreativas em função do uso da substância)? }(\mathrm{n}=246) \\
\text { Apresentou o critério E para síndrome de dependência da CID (abandono de prazeres ou }\end{array}$ & 40,3 & 62,7 & 57,1 & $0,002^{* *}$ \\
\hline interesses alternativos em favor do uso da substância etc.)? $(n=247)$ & 46,6 & 60,3 & 57,1 & 0,064 \\
\hline Foi registrado diagnóstico de comorbidade psiquiátrica? $(n=249)$ & 48,3 & 64,1 & 56,6 & $0,012^{* *}$ \\
\hline \multicolumn{5}{|l|}{ Tratamento anterior ou atual } \\
\hline Já havia, alguma vez, procurado tratamento para alcoolismo? $(\mathrm{n}=284)$ & 47,2 & 60,5 & 54,6 & $0,026^{* *}$ \\
\hline Já havia, alguma vez, procurado tratamento clínico para alcoolismo? $(n=284)$ & 52,3 & 66,0 & 54,6 & 0,086 \\
\hline Já havia, alguma vez, procurado ajuda do $A A ?(n=284)$ & 49,7 & 63,1 & 54,6 & $0,029 * *$ \\
\hline Já havia feito, alguma vez, tratamento ambulatorial em psiquiatria? $(n=282)$ & 49,1 & 61,7 & 54,3 & $0,036^{* *}$ \\
\hline Já havia, alguma vez, sido internado para tratamento por irritabilidade? $(n=117)$ & 63,6 & 38,9 & 59,8 & $0,049^{* *}$ \\
\hline Fez uso de medicação para depressão no mês que precedeu a avaliação? $(n=234)$ & 51,3 & 68,3 & 54,3 & $0,047^{* *}$ \\
\hline Procurou tratamento no PADQ por vontade própria? $(n=285)$ & 62,4 & 49,4 & 54,4 & $0,033^{* *}$ \\
\hline Foi tratado de maneira exclusivamente farmacológica no PADQ? $(n=237)$ & 57,7 & 71,0 & 63,7 & $0,034^{* *}$ \\
\hline
\end{tabular}

* Incluídas todas as variáveis que resultaram p valor <0,1 pelo teste qui-quadrado; ${ }^{* *}$ Resultados plenamente significantes. AA = Alcoólicos Anônimos. 


\section{Características vinculadas ao estado afetivo}

Respostas que se associaram positivamente à adesão foram afirmar sentir-se triste ou sentir-se irritado quando alcoolizado. Sentir-se amedrontado, ameaçado, auto-suficiente, expansivo, insatisfeito, apresentar alguma característica relacionada a bem-estar ou a mal-estar - consideradas em conjunto - quando sóbrio ou sentir-se auto-suficiente, expansivo, conformado quando alcoolizado foram respostas associadas à adesão inferior.

\section{Padrão de consumo}

Beber sozinho por ocasião do início do consumo, beber durante o trabalho por ocasião do início do consumo, beber sozinho por ocasião do aumento do consumo, beber com colegas de trabalho por ocasião do aumento do consumo, beber para enfrentar ou aliviar situações desagradáveis por ocasião do aumento do consumo, consumo ao buscar tratamento de mais que 98 doses da bebida principal e de mais que 154 doses no total, por semana, beber freqüentemente sozinho e costumar dirigir alcoolizado foram padrões de respostas associados à adesão superior. Ter começado a beber com idade acima da média do grupo (16,4 anos), já ter-se envolvido em agressão física com amigos e beber freqüentemente com amigos foram respostas associadas a piores resultados.

\section{Critérios diagnósticos e da comorbidade}

Preencher os critérios 4 e 6 para dependência do DSM-IV isto é, respectivamente, desejo persistente ou esforços para reduzir ou para controlar o uso da substância e abandono ou redução de importantes atividades sociais, ocupacionais ou recreativas em função do uso da substância, preencher o critério E para síndrome de dependência da CID-10 - isto é "abandono de prazeres ou interesses alternativos em favor do uso da substância..." e ter recebido registro de diagnóstico de comorbidade psiquiátrica se associaram positivamente à adesão.

\section{Tratamento anterior ou atual}

Já haver procurado tratamento para alcoolismo, já ter procurado tratamento clínico para alcoolismo, já haver procurado ajuda no alcoólicos anônimos (AA), já haver feito tratamento ambulatorial em psiquiatria, ter feito uso de medicação para depressão no mês que precedeu a avaliação e, também, ter sido tratado de maneira exclusivamente farmacológica no PADQ foram positivamente associados à adesão. Já haver sido, alguma vez na vida, internado para tratamento por irritabilidade e afirmar ter procurado tratamento por vontade própria tiveram associação negativa.

\section{DISCUSSÃO}

Considerados os resultados relativos aos aspectos sociodemográficos avaliados, os achados deste estudo sugerem que pacientes com melhores níveis de relacionamento social - assim entendidos sujeitos com maior estabilidade e/ou responsabilidade em suas relações familiares e de trabalho - aderiram por mais tempo ao tratamento. Pode-se considerar que, em oposição ao trabalho como empregado, o trabalho autônomo implique tanto em laços interpessoais mais frágeis quanto também em dificuldade em manter-se vinculado, por longo prazo, às rotinas de tratamento. Todavia, algumas características incluídas em outros grupos de variáveis também reforçam esta hipótese, entre estas, as associações positivas de adesão superior com início do consumo em período de trabalho, aumento de consumo em conjunto com colegas de trabalho e identificação do problema com o álcool primeiramente pelo patrão foram aqui tomadas como indicativas de melhores níveis de interação social inicial destes sujeitos. A associação dos critérios 6 do DSM-IV e E da CID-10 com maior adesão também foi considerada indicativa de maior vinculação a atividades sociais e de trabalho antes do desenvolvimento da dependência. O fato de dirigir alcoolizado ter sido associado a melhor nível de adesão pode ser conseqüente a maior contato com o dirigir e, portanto, indicador de melhor nível social; contrariamente, a referência a já haver-se envolvido em agressões físicas com amigos - associada negativamente à adesão - foi considerada indicativa de laços interpessoais mais frágeis. Tais achados são coerentes com os de Tucker et al. ${ }^{13}$, que identificaram que, depois das questões de saúde, problemas relacionados a trabalho, relações interpessoais, familiares e atividades sociais estariam entre as razões mais citadas por alcoolistas para interromper o consumo e se manter abstinentes - por extensão, aderir de modo mais completo a tratamentos. Diferentemente, a aceitação do abuso de álcool por parte do ambiente social estaria associada, segundo Vaillant ${ }^{14}$, a maior freqüência de recaídas.

O fato de os pacientes admitirem sentir "algo" ou referirem tremores quando não bebiam no horário habitual associou-se positivamente à adesão superior, da mesma forma que queixas eminentemente físicas - dor, edema dos pés, fraqueza, desmaios e esquecimento - relativas aos 30 dias que antecederam a avaliação. Contudo, sentir-se, especificamente inapetente se não bebia no horário habitual, bem como alguns achados do exame clínico - hipertensão arterial e roncos à ausculta respiratória - se associaram negativamente à adesão. A conclusão de Tucker et al.13 de que os problemas de saúde seriam a razão mais freqüentemente citada para a decisão de se manter abstinente também condiz com a identificação, no presente estudo, de melhores níveis de adesão entre pacientes que referiram mal-estar 
físico tipicamente vinculado ao alcoolismo, como no caso dos tremores de abstinência. Apesar de não ser possível qualquer afirmação definitiva acerca da associação negativa de certos achados do exame clínico com a adesão, tal interação com inapetência quando não bebia foi tomada como decorrente de possível crença, por parte dos sujeitos estudados, de que o "apetite" vinculado ao beber seria positivo à saúde.

Sentimentos observados, quando sóbrios, associaramse de maneira negativa à adesão superior em cinco possibilidades avaliadas - tanto sentimentos habitualmente avaliados quanto bons (auto-suficiente e expansivo), quanto ruins (amedrontado, ameaçado e insatisfeito) - e também em outras duas situações, resultantes, por somatório de dez sentimentos bons ou dez ruins, dos 20 considerados. Quando alcoolizados, a referência a sentimentos caracteristicamente negativos (irritado ou triste) associou-se positivamente à adesão, enquanto sentimentos de tonalidade positiva (expansivo, auto-suficiente, conformado - em oposição a revoltado) se associaram negativamente à adesão superior. Os resultados sugerem que referências a sentimentos de qualquer tonalidade afetiva, quando sóbrios, pode ser preditor de risco para abandono, conforme costuma ser verbalizado pelos próprios alcoolistas, "bebo porque estou alegre, mas bebo também porque estou triste". Por outro lado, os sentimentos após terem bebido parecem separar os sujeitos em dois subgrupos distintos, relativos ao sentir-se bem ou sentir-se mal sob o efeito dos alcoólicos: no primeiro caso, tenderiam ao abandono de tratamento e, no segundo, a melhores níveis de adesão. Ainda que seja necessário diferenciar entre comportamentos de consumo que antecedem ou se sucedem à instalação da síndrome de dependência, os resultados descritos podem ser compreendidos a partir da interação com características de personalidade - como busca do prazer e evitação do sofrimento ${ }^{15,16}$ - ou com fenômenos psicopatológicos agravados ou aliviados pelo uso dos alcoólicos $8,17,18$.

Se, ao início do consumo, variáveis indicativas de boas relações no trabalho se associaram a melhores resultados de adesão, o consumo recente com amigos influenciou negativamente a adesão, de maneira oposta à influência do relato de beber sozinho, associado positivamente à adesão, tanto para o consumo inicial quanto recente. Maior grau de sociabilidade na fase de dependência instalada associou-se, portanto, ao risco de abandono de tratamento. É fato conhecido que maior convívio social, em especial com o mesmo grupo de amigos com os quais se costumava beber, pode, efetivamente, favorecer lapsos e recaídas e, conseqüentemente, o abandono do tratamento. Para Vaillant ${ }^{14}$, os hábitos de beber do grupo social de cada indivíduo afetam significativamente a forma como ele usa ou abusa do álcool. Marlatt e Barrett ${ }^{19}$ referem que a pressão social direta ou indireta seria a segunda situação que com mais freqüência estaria associada a recaídas. Coerentemente, um terço dos pacientes que apresentam lapsos afirma que ver outras pessoas bebendo seria desencadeador do beber ${ }^{20}$.

As crenças relativas aos problemas com o álcool, de algum modo, parecem indicar que assumir relação direta e pessoal sobre o problema conduziria a melhores resultados terapêuticos: a afirmação de problemas psicológicos e de saberem por que consumiam álcool - associadas a mais longa adesão - sugere percepção de responsabilidade pessoal relativa ao consumo de alcoólicos, enquanto a afirmação de necessidade de desintoxicação poderia indicar projeção desta responsabilidade sobre o serviço terapêutico, com influência negativa sobre a adesão. A associação positiva entre adesão superior e o registro do critério 4 para dependência do DSM-IV também parece reforçar tal hipótese. Efetivamente, auto-eficácia mais elevada, maior envolvimento com o programa de tratamento e maior percepção do tratamento, como forma de ajuda, associam-se a melhores resultados terapêuticos ${ }^{21}$. O encontro de piores taxas de adesão entre aqueles que procuraram o tratamento por vontade própria aponta para a influência de fatores mais complexos; e os resultados contraditórios relativos à influência da própria família também sugerem relação ambivalente dos sujeitos com seu núcleo familiar. Nos limites deste trabalho não é possível responder com clareza a estas questões; todavia, deve-se ressaltar que outros pesquisadores têm obtido resultados controversos quanto à relação entre fontes de encaminhamento e resultados de tratamento: Martin et al. ${ }^{5}$ registraram que sujeitos em tratamento em razão de mandato judicial tiveram melhor adesão que os voluntários. Callaghan e Cunningham ${ }^{22}$ também apontaram maiores taxas de abandono entre aqueles que tinham procurado tratamento por vontade própria. Atkinson et al. ${ }^{23}$, por sua vez, concluíram que pacientes encaminhados por problema legal ou por influência da família/iniciativa própria tiveram melhor resultado que os encaminhados por serviços de saúde ou serviço social.

As referências a já ter, alguma vez na vida, procurado algum tratamento para alcoolismo - seja clínico, ajuda do AA ou não-especificado -, já haver feito tratamento ambulatorial em psiquiatria e ter feito uso de medicação para depressão no mês anterior à avaliação influenciaram positivamente à adesão; já haver sido internado para tratamento de irritabilidade teve influência inversa sobre a adesão. Efetivamente, tratamento prévio ${ }^{23}$, maior número de internações para desintoxicação ${ }^{6}$, assim como maior freqüência ao $A A^{6,24}$ já foram identificados como preditores de bons resultados de tratamento. Por outro lado, pode-se supor que internações decorrentes de episódios de irritabilidade teriam sido buscadas por terceiros - em circunstâncias 
de ausência de autocrítica; a consciência da própria irritabilidade sob efeito do álcool - conforme referido anteriormente, na abordagem de sentimentos - resultaria mais alto nível de insight e, por conseguinte, de adesão ao tratamento.

Merece ainda consideração o fato e, neste estudo, baixa idade de início de consumo, padrão de consumo recente mais elevado e registro de comorbidade psiquiátrica - características clínicas habitualmente vinculadas a subtipos de maior gravidade - se associaram positivamente à adesão. Deve-se ter em mente que, coerentemente com os critérios de encaminhamento ao PADQ, 93,8\% dos sujeitos aqui estudados receberam diagnóstico de síndrome de dependência ao álcool e 93,5\% apresentavam evidências de dependência física de alcoólicos. Tais percentuais tornam evidente a maior gravidade clínica - ao menos quanto ao diagnóstico de alcoolismo - desta população diante dos grupos de pacientes que costumam ser apresentados e discutidos por outros autores. Ainda que diferentes estudos tenham demonstrado que a maior parte das pessoas com dependência de álcool apresentaria alguma comorbidade psiquiátrica associada, costuma-se enfatizar o elevado percentual de sujeitos com transtorno de personalidade anti-social (TPAS) nas amostras estudadas. Muito embora se acentue a relevância dos TPAS na abordagem do alcoolismo - associando-os a maior gravidade dos problemas relacionados ao álcool e piores resultados do tratamento ${ }^{25-}$ 28 -, neste estudo, $52,6 \%$ dos pacientes tiveram registro de alguma comorbidade psiquiátrica e apenas um sujeito recebeu o diagnóstico de TPAS.

Neste sentido, considera-se que as comorbidades apresentadas pelos pacientes incluídos neste estudo tenham favorecido a adesão, independentemente de serem primárias ou secundárias ao alcoolismo. Tal fato é consistente com o achado de maiores taxas de adesão superior entre os pacientes que foram abordados por modalidades farmacológicas; como, habitualmente, no PADQ, a indicação de tratamento exclusivo pelo uso de psicofármacos se fazia em virtude de comorbidades-alvo, os resultados sugerem que a prescrição exclusiva tenha sido, de fato, relevante para o resultado do tratamento. Ou seja, ao focarem comorbidades responsivas ao seu uso, os psicofármacos aliviavam sintomas cuja presença poderia conduzir à recaída, contribuindo, assim, para a melhor adesão ao tratamento. Ainda que não se possa descartar a possibilidade de que a exigência de presença mais freqüente ao serviço, por parte de pacientes aos quais se indicou intervenção simbólica, estes resultados convergem para os achados de Long et al. ${ }^{21}$, no sentido de que a redução de sintomas se associaria a melhores resultados do tratamento.

Tudo considerado, os resultados deste estudo reforçam a hipótese de que o tempo de adesão constitui alternativa adequada para a avaliação de tratamento de alcoolistas e de fatores relacionados a melhores ou piores resultados, em especial quando comparado a outras medidas de maior complexidade e de mais difícil mensuração.

\section{Limitações e pontos fortes do estudo}

Entre as limitações deste estudo - como de todos os que utilizam semelhante desenho - ressalta-se a reduzida possibilidade de generalização dos resultados, uma vez que a admissão dos sujeitos obedece, tão-somente, a considerações clínico-administrativas associadas à política assistencial adotada, sem qualquer outro critério de seleção. A simplicidade de seu desenho, porém, permite que ele possa ser facilmente reproduzido em diferentes contextos assistenciais.

A exclusão dos pacientes que não completaram a fase de avaliação poderia, em princípio, levar ao questionamento da representatividade do grupo avaliado. Todavia, devese considerar que, na maior parte dos estudos, critérios de inclusão e exclusão bastante estritos costumam excluir do tratamento sujeitos que, de início, desejavam tratar-se; tais restrições geram outros vieses de seleção.

O elevado número de pacientes incluídos neste estudo dificilmente é encontrado em estudos brasileiros e devese considerar ainda que estudos que se desdobram a partir de circunstâncias de "vida real" têm sido cada vez mais valorizados, exatamente por possibilitarem importantes discussões acerca de aspectos relativos à efetividade das práticas assistenciais ${ }^{3,29}$.

\section{CONCLUSÕES}

A associação - positiva ou negativa - de diferentes características dos pacientes ao tempo de adesão ao tratamento reforça a necessidade de aprofundamento de estudos que facilitem o processo de avaliação, tanto de pacientes quanto de resultados de tratamentos. Tal perspectiva de pesquisa visa, em última instância, a contribuir para o desenvolvimento de estratégias diferenciadas para o cuidado de determinados grupos de pacientes que costumam responder mal a abordagens terapêuticas de rotina. Em especial, o fato de terem sido identificados grande número de variáveis que não se vincularam estatisticamente à adesão dos pacientes poderá, no futuro, contribuir para a simplificação das rotinas de avaliação de alcoolistas. O aumento do número de sujeitos avaliados e a utilização de procedimentos de análise multivariada poderão gerar resultados ainda mais consistentes.

Conflito de interesses: $O$ artigo não está limitado por conflitos de interesses. MAG, GFS e RBN são bolsistas BIC-UFJF. KDCS é bolsista PROVOQUE-PIBIC. 


\section{REFERÊNCIAS}

1. Finney JW. Assessing treatment and treatment processes. In: Allen JP, Wilson VV, editores. Assessing alcohol problems: a guide for clinicians and researchers. 2. ed. Bethesda: US Department do Health and Human Services/National Institute on Alcohol Abuse and Alcoholism; 2003.

2. Connors GJ, Walitzer KS, Dermen, KH. Preparing clients for al coholism treatment: effects on treatment participation and outcomes. preparing clients for alcoholism treatment: effects on treatment participation and outcomes. J Consult Clin Psychol. 2002;70(5):1161-9.

3. Corrao G, Bagnardi V, Zambon A, Aricò S, Dall'Aglio C, Addolorato G. Outcome variables in the evaluation of alcoholics' treatment: lessons from the italian assessment of alcoholism treatment (ASSALT) project. Alcohol Alcohol. 1999;34(6):873-81.

4. Landau J, Stanton MD, Brinkman-Sull D, Ikle D, McCormick D, Garrett J, et al. Outcomes with the ARISE approach to engaging reluctant drug- and alcohol-dependent individuals in treatment. Am J Drug Alcohol Abuse. 2004;30(4):711-38.

5. Martin BK, Clapp L, Alfers J, Beresford TP. Adherence to court-ordered disulfiram at fifteen months: a naturalistic study. J Subst Abuse Treat. 2004;26(3):233-36.

6. Timko C, Moos RH, Finney J, Connell EG. Gender differences in help-utilization and the 8-year course of alcohol abuse. Addiction. 2002;97(7):877-89.

7. Oslin DW, Pettinati H, Volpicelli JR. J Alcoholism treatment adherence: older age predicts better adherence and drinking outcomes. Geriatr Psychiatry. 2002:10:740

8. Rounsaville BJ, Dolinsky ZS, Babor TF, Meyer RE. Psychopathology as a predictor of treatment outcome in alcoholics. Arch Gen Psychiatry. 1987;44: 505-13.

9. Ribeiro MS, Alves MJM, Guirro UBP, Baldi BG. Alcoolismo: a influência do reconhecimento da comorbidade na adesão de pacientes ao programa terapêutico. J Bras Psiquiatr. 2004:53(2):124-32.

10. Moos RH, Moos BS. Long-term influence of duration and intensity of treatment on previously untreated individuals with alcohol use disorders. Addiction. 2003;98(3):325-38.

11. Moyer A, Finney JW, Swearingen CE, Vergun P. Brief interventions for alcohol problems: a meta-analytic review of controlled investigations in treatment-seeking and non-treatmentseeking-populations. Addiction. 2002;97(3):279.

12. O'Connor SM, Davies JB, Heffernan DD, van Eijk R. Alternative method for predicting attrition from an alcohol treatment programme. Alcohol Alcohol. 2003;38(6):568-73.

13. Tucker JA, Vuchinich RE, Rippens PD. Environmental contexts surrounding resolution of drinking problems among problem drinkers with different help-seeking experiences. J Stud Alcohol. 2002;63(3):334-41.

14. Vaillant GE. A história natural do alcoolismo revisitada. Porto Alegre: ArtMed Press; 1999
15. MacAndrew C. What the MAC tells us about men alcoholics: An interpretative review. Q J Stud Alcohol. 1981;42:604-25.

16. Wolf AW, Schubert DSP, Patterson M, Grande T, Pendleton L. The use nof the MacAndrew scale in detecting substance abuse and antisocial personality. J Pers Assess. 1990;54(3/4):747-55.

17. Driessen M, Veltrup C, Wetterling T, John U, Dilling H. Axis I and Axis II comordity in alcohol dependence and the two types of alcoholism. Alcohol Clin Exp Res. 1998;22(1):77-86.

18. Morgenstern J, Langenbucher J, Labouvie E, Miller KJ. The comorbidity of alcoholism and personality disorders in a clinical population: prevalence rates and relation to alcohol typology variables. J Abnorm Psychol. 1997;106(1):74-84.

19. Marlatt GA, Barrett K. Prevención de recaídas. In: Galanter M, Kleber HD, editores. Tratamiento de los trastornos por abuso de sustancias de la american psychiatric. Barcelona: Masson Press; 1997. p. 291-305.

20. Blow FC, Walton MA, Chermack ST, Mudd SA, Brower KJ. Older adult treatment outcome following elder-specific inpatient alcoholism treatment. J Subst Abuse Treat. 2000;19:67-75.

21. Long CG, Williams M, Midgley M, Hollin CR. Within-program factors as predictors of drinking outcome following cognitive-behavioral treatment. Addict Behav. 2000;25(4):573-8.

22. Callaghan RC, Cunningham JA. Gender differences in detoxification: predictors of completion and re-admission. J Subst Abuse Treat. 2002;23(4):399-407.

23. Atkinson RM, Misra S, Ryan SC, Turner JA. Referral paths, patient profiles and treatment adherence of older alcoholic men. J Subst Abuse Treat. 2003;25:29-35.

24. Gossop M, Harris J, Best D, Man LH, Manning V, Marshall J, et al. Is attendance at al coholics anonymous meetings after inpatient treatment related to improved outcomes? A 6-month follow-up study. Alcohol Alcohol. 2003;38(5):421-6.

25. Anthenelli RM, Smith TL, Irwin MR, Schuckit MA. A Comparative study of criteria for subgrouping alcoholics: the primary/secundary diagnostic scheme versus variations of the type 1/type 2 criteria. Am J Psychiatry. 1994;151:1468-74.

26. Sannibale C, Hall W. An evaluation of Cloninger's typology of alcohol abuse. Addiction. 1998:93(8):1241-9.

27. von Knorring L, Von Knorring A-L, Smigan L, Lindberg ULF, Edholm M. Personality traits in subtipes of alcoholics. J Stud Alcohol. 1987:48(6):523-7.

28. Hesselbrock VM, Hesselbrock MN. Are there empirically supported and clinically useful subtypes of alcohol dependence? Addiction. 2006;101(1):97-103.

29. Haro JM, Suarez D, Novick D, Usall J, Naber D. Three-year antipsychotic effectiveness in the outpatient care of schizophrenia: Observational versus randomized studies results. Eur Neuropsychopharmacol. 2006;17(4):235-44. 